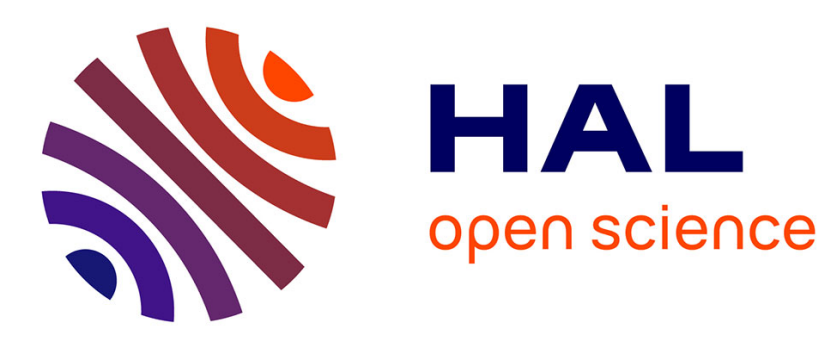

\title{
IVIg for relapsing-remitting multiple sclerosis: promises and uncertainties
}

\author{
Jagadeesh Bayry, Hans-Peter Hartung, Srini V Kaveri
}

\section{To cite this version:}

Jagadeesh Bayry, Hans-Peter Hartung, Srini V Kaveri. IVIg for relapsing-remitting multiple sclerosis: promises and uncertainties. Trends in Pharmacological Sciences, 2015, 36 (7), pp.419-421. 10.1016/j.tips.2015.04.012 . hal-01155485

\section{HAL Id: hal-01155485 \\ https://hal.sorbonne-universite.fr/hal-01155485}

Submitted on 26 May 2015

HAL is a multi-disciplinary open access archive for the deposit and dissemination of scientific research documents, whether they are published or not. The documents may come from teaching and research institutions in France or abroad, or from public or private research centers.
L'archive ouverte pluridisciplinaire HAL, est destinée au dépôt et à la diffusion de documents scientifiques de niveau recherche, publiés ou non, émanant des établissements d'enseignement et de recherche français ou étrangers, des laboratoires publics ou privés. 


\section{FORUM}

\section{IVIg for relapsing-remitting multiple sclerosis: promises and uncertainties}

\section{Jagadeesh Bayry $^{1,2,3,4,5}$, Hans-Peter Hartung ${ }^{6}$, Srini V Kaveri ${ }^{1,2,3,4,5}$}

${ }^{1}$ Institut National de la Santé et de la Recherche Médicale Unité 1138, Paris, F-75006, France

${ }^{2}$ Centre de Recherche des Cordeliers, Equipe - Immunopathology and therapeutic immunointervention, Paris, F-75006, France

${ }^{3}$ Sorbonne Universités, UPMC Univ Paris 06, UMR S 1138, Paris, F-75006, France

${ }^{4}$ Université Paris Descartes, Sorbonne Paris Cité, UMR S 1138, Paris, F-75006,

France

${ }^{5}$ International Associated Laboratory IMPACT (Institut National de la Santé et de la Recherche Médicale, France - Indian Council of Medical Research, India), National Institute of Immunohematology, Mumbai, 400012, India

${ }^{6}$ Department of Neurology, Medical Faculty, Heinrich-Heine-University, Moorenstr. 5, D-40225 Düsseldorf, Germany

Corresponding author: Jagadeesh Bayry, Institut National de la Santé et de la Recherche Médicale Unité 1138, Equipe - Immunopathology and therapeutic immunointervention, Centre de Recherche des Cordeliers, 15 rue de l'Ecole de Médicine, Paris, F-75006, France. Tel: 00331442782 03; Fax: 0033144278194.

E-mail: jagadeesh.bayry@crc.jussieu.fr 


\begin{abstract}
Despite promising clinical trials, intravenous immunoglobulin (IVIg) therapy in relapsing-remitting multiple sclerosis has met with uncertainties that might be attributed to small patient cohorts, heterogeneity in the patients, dosage of IVIg, and the duration and window of treatment.
\end{abstract}

Key words: IVIg, multiple sclerosis, biomarkers, immunotherapy, autoimmunity, inflammation 
Multiple sclerosis (MS) is the most common chronic inflammatory disease of the central nervous system (CNS). Aberrant immune responses in patients lead to demyelination and axonal degeneration, causing accumulated disability and reduced life expectancy[1]. The disease is highly heterogeneous, and the majority of patients are initially diagnosed with relapsing-remitting multiple sclerosis (RRMS). RRMS is characterized by worsening of neurological symptoms followed by remission that may be complete initially but only partial as the disease advances. Although new drugs such as natalizumab, fingolimod, teriflunomide, dimethylfumarate and alemtuzumab have been recently added to the traditional therapeutic armamentarium of interferon $\beta$, glatiramer acetetate and mitoxantrone, safety issues continue, particularly during pregnancy and lactation periods and long-term use, which keeps clinicians looking for alternative therapies.

\section{The basis for IVIg therapy in RRMS}

Intravenous immunoglobulin (IVIg), a pooled preparation of normal IgG obtained from the plasma of several thousand healthy donors was initially used for the replacement therapy of primary and secondary immunodeficiencies. Seminal work on therapeutic utility of IVIg in the treatment of immune thrombocytopenia by Imbach in 1981 encouraged exploration of IVIg therapy in a number of conditions considered to be autoimmune in nature. Subsequently, IVIg has been established as first-line therapy for many autoimmune and inflammatory conditions including Guillain-Barré syndrome and chronic inflammatory demyelinating polyradiculoneuropathy.

Based on observations in basic research, animal models and patient studies, it was proposed that IVIg provides benefit to patients with autoimmune disorders via several mutually nonexclusive mechanisms: inhibition of activation of macrophages, 
dendritic cells and pathogenic $\mathrm{T}$ cells such as Th1 and Th17 cells; expansion of regulatory $\mathrm{T}$ cells; modulation of $\mathrm{B}$ cell responses and inhibition of pathogenic autoantibody production; suppression of production of inflammatory cytokines and stimulation of anti-inflammatory cytokines; neutralization of pathogenic autoantibodies via anti-idiotypes and inhibition of activation of endothelial cells and complement[2]. These data, a proven track record of minimal side effects in treated patients and promising results obtained with experimental models of MS and several case reports prompted the initiation of clinical trials for exploring utility of IVIg therapy in RRMS (Figure 1).

\section{Initial positive clinical trials}

The initial four clinical studies from Europe showed beneficial effects of IVIg for RRMS. In the Austrian study, patients received monthly doses of 0.15 to $0.2 \mathrm{~g} / \mathrm{kg}$ for the period of 2 years[3]. This randomized, double-blind, placebo-controlled, multicentre study reported that monthly IVIg infusion is beneficial to reduce the evolution of further clinical disability as measured by the absolute change in Kurtzke's expanded disability status scale score (EDSS).

In 1998 two studies reported benefits of IVIg for RRMS[4, 5]. The study from Israel was randomized, double-blind and placebo-controlled [4]. The dose of IVIg was $0.4 \mathrm{~g} / \mathrm{kg}$ over five days and followed by $0.4 \mathrm{~g} / \mathrm{kg}$ for every two months for two years. IVIg-treated patients showed significant reduction in yearly exacerbation rate while six patients were completely relapse-free. Also, the median time to first exacerbation was three times higher in the IVIg group. Although analysis of total lesions by brain magnetic resonance imaging (MRI) did not reveal significant differences between two groups, this study suggested that IVIg is effective in reducing relapse rate in RRMS. 
The Danish study was a randomized, double-blind, crossover study [5]. One group was treated with IVIg on a monthly basis for six months at $1 \mathrm{~g} / \mathrm{kg}$ given on two consecutive days. After three months of washout period, patients received placebo for 6 months. The second group was treated in reverse order. The number of exacerbation-free patients was significantly higher during IVIg treatment. Also, serial MRI revealed significantly fewer enhancing lesions per patient/scan during IVIg treatment. A double-blind, placebo-controlled study in Poland reported that IVIg dose $0.2 \mathrm{~g} / \mathrm{kg}$ administered once a month for 12 months is equally effective as $0.4 \mathrm{~g} / \mathrm{kg}$ in reducing MS activity[6]. Subsequent data from a retrospective multicenter observational study over five years reported that IVIg treatment $(0.24 \pm 0.15 \mathrm{~g} / \mathrm{kg} / \mathrm{month})$ resulted in $69 \%$ reduction in mean annual relapse rate[7]. Collectively, these studies suggested that IVIg is effective in reducing relapse rate in RRMS.

Due to safety issues of currently licensed MS drugs during pregnancy and lactation periods, as a retrospective study, IVIg was explored in pregnant patients with RRMS[8]. Patients received IVIg for the whole pregnancy $(0.4 \mathrm{~g} / \mathrm{kg} / \mathrm{day}$ for 5 consecutive days within first 6-8 weeks of pregnancy followed by $0.4 \mathrm{~g} / \mathrm{kg} / \mathrm{day}$ every 6 weeks until 12 weeks post-partum) had significantly diminished relapse rate/woman/year as compared to untreated patients. Another multinational, multicentre, randomized double-blind clinical trial also supported usefulness of IVIg in postpartum RRMS[9]. 


\section{The negative data}

The excitement emanating from these successful trials receded when a subsequent multi-center, randomized, double-blind, placebo-controlled trial PRIVIG reported ineffectiveness of IVIg for RRMS[10]. This study involving two doses of IVIg (0.2 and $0.4 \mathrm{~g} / \mathrm{kg}$ every 4 weeks for 12 months) demonstrated that the proportion of relapse-free patients and cumulative number of unique newly active MRI lesions did not differ among IVIg-treated groups and placebo.

\section{Reasons for uncertainties}

While many auto-inflammatory diseases have proven responsive to IVIg therapy, the outcome of these MS trials remains somewhat of a conundrum. Several factors might have contributed to the equivocal observations. Foremost, the heterogeneous nature of the disease and its underlying pathobiology may be confounding factors. In all these clinical trials, patients groups were not uniform and cohorts were relatively small (Figure 1).

Another important reason for uncertainties with these trials is the dosage, window and duration of the IVIg treatment employed. At least four different dose regimens were used in the above studies. Currently, high-dose IVIg therapy, typically $1-2 \mathrm{~g} / \mathrm{kg}$ infused as either single dose or over two or five consecutive days, is commonly used to treat autoimmune and inflammatory diseases. Two of the clinical trials and a retrospective study in pregnant patients with RRMS that reported beneficial effects of IVIg had adopted this dose regimen. However, in other studies including the PRIVIG study [10], the dose of IVIg administered to the patients correspond to replacement doses used in primary and secondary immunodeficiencies $(0.4 \mathrm{~g} / \mathrm{kg}$ every four weeks) 
or were even lower. Thus, cumulative dose of IVIg used in the PRIVIG study might have been too low to be efficacious (Figure 1).

\section{The next steps}

One way to deal with the challenge of disease heterogeneity would be the identification of biomarkers associated with therapeutic responsiveness to IVIg in an individual patient. Functional genomics has been recently performed to predict IVIg response in RRMS patients[11]. This GAM-25 study indicates that a combination of genotyping and 65 functional immune parameters such as modulation of Fc-receptors, cytokines, chemokines and natural killer cells subjected to a stepwise linear discriminant analysis, could be used to distinguish patients who benefit from IVIg therapy from those who do not. Applying a scoring system based on these parameters, it was suggested that RRMS patients with mild disease respond to IVIg therapy. The cohort enrolled in this study was small, without control group and hence data were only suggestive. However, this study should form a platform for a validation trial involving more patients. Importantly, a prospective, multi-centre, active-controlled, randomized Phase IIIb trial is underway with a larger number of patients by the same authors (http://www.isrctn.com/ISRCTN82177408). As the GAM-25 study utilized an IVIg dose that corresponds to replacement therapy $(0.4 \mathrm{~g} / \mathrm{kg}$ for every four weeks until study termination), clinical trials should be considered with high-dose IVIg (1-2 $\mathrm{g} / \mathrm{kg}$ ) therapy as well. The molecular and immunological biomarkers that were used in other autoimmune patients to segregate IVIg responders could also be tested in future trials[12]. If subtype, activity and response marker studies yield conclusive results that predict which patients will profit from IVIg, a safe new addition to the therapeutic armamentarium to battle this crippling disease would be available. It is hoped that 
IVIg then could be optimized and streamlined with precise anti-inflammatory dosage, optimal window of treatment and duration of therapy.

\section{Acknowledgments}

Supported by Institut National de la Santé et de la Recherche Médicale (INSERM), Centre National de la Recherche Scientifique (CNRS), Université Pierre et Marie Curie and Université Paris Descartes. (Dr. Bayry and Dr. Kaveri); James und Elisabeth Cloppenburg Stiftung Düsseldorf and Walter und Ilse-Rose-Stiftung (Dr. Hartung). Because of space limitations, we could only refer to key reports. This of course does not lessen the great value of studies not quoted.

\section{Conflict of interests}

Dr Bayry and Dr Kaveri received research funding from CSL Behring, Grifols, Laboratoire Français du Fractionnement et des Biotechnologies. Dr. Hartung received fees for consulting, speaking and serving on steering committees, with approval by the Rector of Heinrich-Heine-University, from Bayer Healthcare, Baxter, Biogen Idec, CSL Behring, GeNeuro, Genzyme, Grifols, MedImmune, Merck Serono, Novartis, Octapharma, Opexa, Receptos, Teva, Sanofi. 


\section{References}

1. Compston, A. and Coles, A. (2008). Multiple sclerosis. Lancet 372, $1502-$ 1517

2. Buttmann, M. et al. (2013). Polyclonal immunoglobulin G for autoimmune demyelinating nervous system disorders. Trends Pharmacol. Sci. 34, 445-457

3. Fazekas, F. et al. (1997). Randomised placebo-controlled trial of monthly intravenous immunoglobulin therapy in relapsing-remitting multiple sclerosis. Austrian Immunoglobulin in Multiple Sclerosis Study Group. Lancet 349, $589-593$

4. Achiron, A. et al. (1998). Intravenous immunoglobulin treatment in multiple sclerosis. Effect on relapses. Neurology 50, 398-402

5. Sorensen, P.S. et al. (1998). Intravenous immunoglobulin G reduces MRI activity in relapsing multiple sclerosis. Neurology 50, 1273-1281

6. Lewanska, M, et al (2002). No difference in efficacy of two different doses of intravenous immunoglobulins in MS: clinical and MRI assessment. Eur. J. Neurol. 9, 565-572

7. Haas, J. et al. (2005). Intravenous immunoglobulins in the treatment of relapsing remitting multiple sclerosis-results of a retrospective multicenter observational study over five years. Mult. Scler. 11, 562-567

8. Achiron, A. et al. (2004). Effect of intravenous immunoglobulin treatment on pregnancy and postpartum-related relapses in multiple sclerosis. J. Neurol. $251,1133-1137$

9. Haas, J. and Hommes, O.R. (2007). A dose comparison study of IVIG in postpartum relapsing-remitting multiple sclerosis. .Mult. Scler. 13, 900-908 
10. Fazekas, F. et al (2008). Intravenous immunoglobulin in relapsing-remitting multiple sclerosis: a dose-finding trial. Neurology 71, 265-271

11. Berger, T. et al. (2014). Predicting therapeutic efficacy of intravenous immunoglobulin (IVIG) in individual patients with relapsing remitting multiple sclerosis (RRMS) by functional genomics. J. Neuroimmunol. $277,145-152$

12. Galeotti, C. et al. (2015). Molecular and immunological biomarkers to predict IVIg response. Trends Mol. Med. 21, 145-147 


\section{Figure legend}

Figure 1. The promises and uncertainties of IVIg therapy for relapsing-remitting multiple sclerosis (RRMS). The rationales for the IVIg therapy in RRMS are presented on the left side ('happy face'), while the possible reasons for uncertainties with IVIg therapy are depicted on the right side ('sad face').

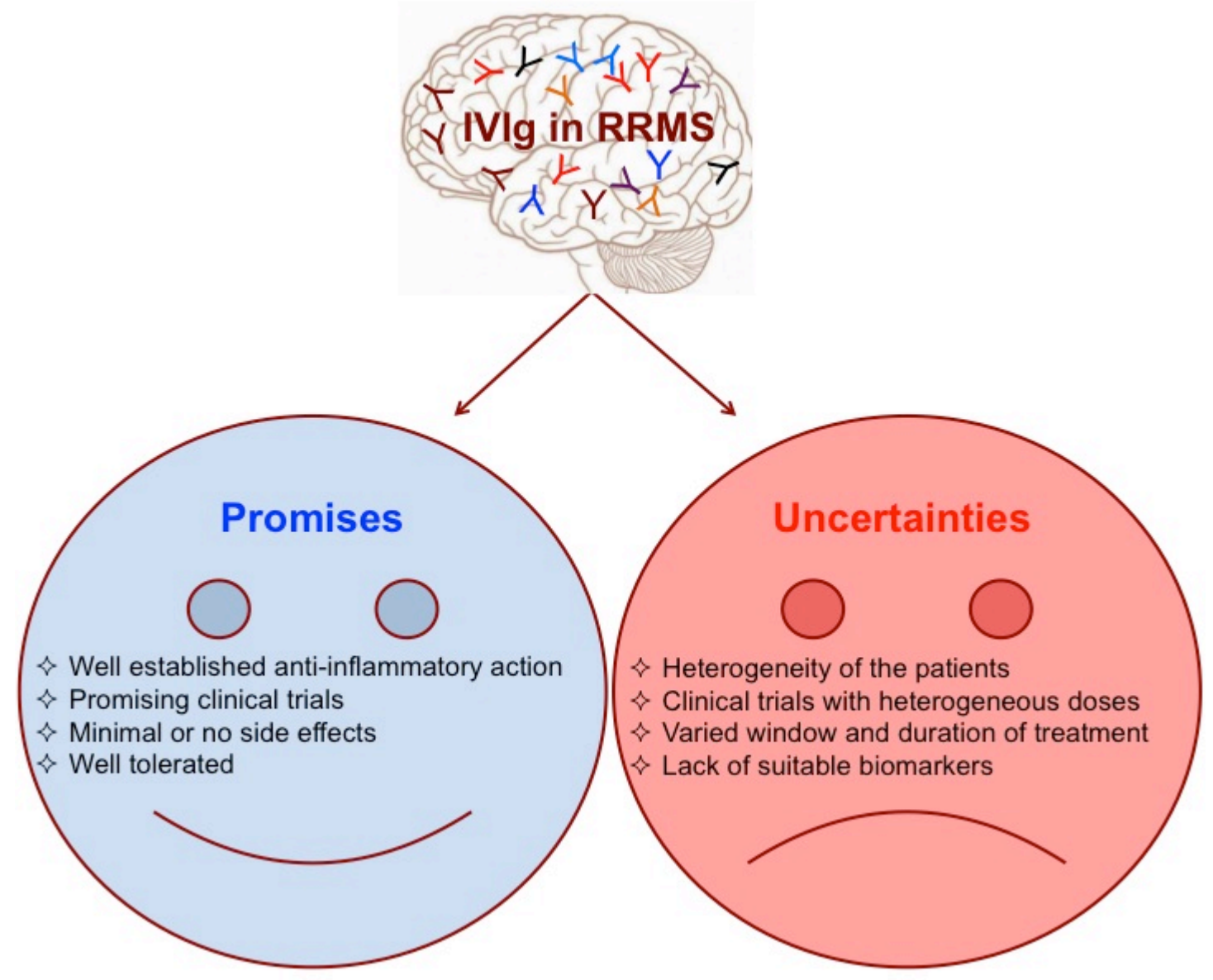

